\title{
Virtually Renovating the Trauma Film Paradigm: Comparing Virtual Reality With On-Screen Presentation of an Analogue Trauma
}

\author{
Grace Baptie, Jackie Andrade, Alison M. Bacon, \& Alyson Norman \\ University of Plymouth, Plymouth, United Kingdom
}

\begin{abstract}
Due to the unpredictable nature of traumatic events, prospective research into trauma relies upon laboratory methods utilising distressing film scenes to act as a trauma analogue measuring vulnerability factors and testing interventions applied to posttraumatic stress disorder (PTSD). This is the first study to test whether Virtual Reality (VR) provides a more effective trauma analogue than traditional on-screen presentation by direct comparison of the same real-life trauma film. Participants viewed footage of a staged car accident either presented in VR $(N=31)$ or on-screen (OS) ( $N=30)$. Both groups recorded sense of presence, pre- and post-film mood and state anxiety. After the film, some participants (VR: $n=18$; OS: $n=12$ ) reported involuntary intrusions of the film and recorded the emotionality of these. VR presentation evoked a greater sense of presence, yet both VR and OS presentation elicited negative mood and involuntary intrusions. Although intrusions were more vivid in the VR condition, there were no significant differences in frequency or distress. However, a greater sense of presence, regardless of medium, was predictive of increased emotional reaction to the film and greater intrusion frequency and distress. Therefore, implementing a VR paradigm could be directly beneficial for TFP research concerning sense of presence or vividness of intrusions. The association between enhanced sense of presence and stress response suggests that an effective trauma analogue should be immersive, and VR presentation is a useful medium to elicit a greater sense of immersion.
\end{abstract}

Keywords: Trauma Film Paradigm; virtual reality; involuntary memory; intrusions; immersion

\section{Introduction}

Experiencing intrusive images or thoughts after a traumatic event is a common feature of post-trauma cognition. Prospective trauma research, which utilises a film showing stressful content as an analogue for a traumatic event, typically record post-film intrusive imagery and thoughts as a measure of trauma response (Horowitz, 1969). The use of a traumatic film as an analogue for a trauma event has particular relevance due to the $5^{\text {th }}$ edition of the Diagnostic and Statistical Manual of Mental Disorders (DSM-5) incorporating exposure to trauma through electronic media, TV, movies or pictures as part of the classification for posttraumatic stress disorder (PTSD) (American Psychiatric Association, 2013). Incorporating a trauma film also circumvents methodological drawbacks concerning retrospective trauma research that relies on victims' memories of traumatic events that may have occurred many years previously (Candel \& Merckelbach, 2004). Alternatively, the 'Trauma Film Paradigm' (TFP) provides a platform to prospectively research trauma in a controlled setting (Horowitz, 1969). The paradigm involves healthy participants completing pre-film measures before watching a short film depicting stressful events, which acts as an analogue for a trauma incident. Following this, participants complete post-film measures of emotional response and record any involuntary intrusions experienced as a result of the film in an intrusion diary over the following few days. The TFP has been shown to elicit acute responses in healthy participants akin to those 
experienced after actual trauma exposure, for example, heightened physiological arousal (Chou et al., 2014); increased state anxiety and rumination, (Laposa \& Rector, 2012) avoidance behaviour (Halligan et al., 2002) and later intrusive memories of the trauma scenes (Holmes et al., 2004).

The qualitative similarities of symptoms following both direct exposure and analogue trauma means that the TFP can provide a relevant and worthwhile medium to prospectively measure psychological responses to trauma and test the effectiveness of interventions to combat intrusion symptoms within clinical PTSD (lyadurai et al., 2018). In traditional film paradigms the observer remains an 'outsider', whereas Virtual Reality (VR) can offer an extension to TFP research by creating a more immersive environment with increased participant interactivity and realism (Dibbets \& Schulte-Ostermann, 2015). In comparison to viewing a film on-screen, VR is associated with greater immersion and sense of presence (Riva et al., 2007) and offers a first-person perspective and person-environment interaction, enabling the generation of personal autobiographical memories (Schöne et al., 2019). VR has already been effectively incorporated into exposure-based therapy (VRET) for the treatment of PTSD, (Beidel et al., 2019; Botella et al., 2015; Loucks et al., 2019) and has yielded equal treatment results to in vivo exposure (Carl et al., 2019). Virtual environments are also used in pre-deployment military training of stress resilience and emotional coping (Rizzo et al., 2012), yet the use of VR in TFP research is a relatively recent innovation.

Perhaps one explanation for the scarcity in VR TFP research concerns the unique filming method required to produce VR films. Realistic $360^{\circ}$ film footage as an appropriate analogue for a real-life trauma event is difficult to attain. As a result, VR studies typically rely on programmed animations of simulated stressful environments such as an animation of the aftermath of a train collision (Boskovic et al., 2019; Dibbets, 2020) or simulated bomb detonation (Schweizer et al., 2017; Schweizer et al., 2019). Such VR scenes have been found to increase physiological response (Schweizer et al., 2018) and evoke intrusions later (Cuperus et al., 2016; Dibbets, 2020). Yet without a direct comparison with on-screen presentation, it is unclear whether the benefit of increased immersion from VR outweighs the potential costs in realism from using an animated analogue trauma. Recent imagery research has alluded to multisensory VR as a more effective medium to present a trauma analogue when compared with multisensory script-driven imagery, induced from an analogue trauma presented via audio script with olfactory stimulation (Schweizer et al., 2018). This emphasises the potential benefits of providing a multisensory virtual environment in TFP research, at least when compared with multisensory script-driven imagery. However very few studies have endeavoured to draw direct comparisons between VR and on-screen presentation of a stressful film to elucidate the true value of renovating traditional TFP methodology with a more immersive analogue trauma in VR.

The first study comparing traditional on-screen TFP with a VR alternative was conducted by Dibbets and SchulteOstermann (2015). A clip from a feature film presented on screen displaying an acted domestic violence scene was compared with a programmed animated depiction of a domestic assault in VR. The VR scene was found to evoke a greater sense of immersion, yet this did not translate to a higher frequency of intrusions or stronger trauma response, conversely the on-screen film resulted in greater distress elicited from intrusions. The study provides useful preliminary insights into virtual trauma analogues, yet the disparity between trauma films makes it difficult to ascertain whether these findings bear a true reflection of the effectiveness of incorporating VR into TFP research or a reduced trauma response to an animated virtual environment. A second comparable study progressed with the addition of participant engagement and interactivity within the virtual environment (Cuperus et al., 2017). Acted scenes of violence presented on-screen were compared with an interactive programmed virtual environment involving participants navigating an animated fantasy horror scene in VR. Similarly, the VR game was reported to be more personally involving yet did not elicit a greater frequency of intrusions when compared with the on-screen film. In contrast, participants who viewed the violent film on-screen reported greater unpleasantness from intrusions.

These foundational studies are useful in providing a basis for VR paradigms to be utilised in prospective TFP research and imply greater immersion from VR but no more traumatic, as defined by intrusion frequency, than on-screen presentation. The relevance of immersion is particularly relevant in TFP research due to the bidirectional relationship between affective response and feeling of presence associated with virtual environments (Riva et al., 2007). Therefore, these studies provide some preliminary insights into the potential value of renovating the traditional paradigm using VR but have some methodological shortcomings when attempting to draw direct comparisons between on-screen and virtual presentation of a trauma analogue. 
Namely, the reliance on programmed animated scenes leading to inconsistency between films used in on-screen and VR conditions and possible issues with realism when regarded as an analogue for a real-life trauma event. The credibility of a virtual scene, referring to the extent the film fits with reality, is a fundamental factor in generalising TFP research to experiences of real life trauma (Rovira et al., 2009). Consequently, current literature would benefit from a direct comparison of the same realistic trauma film, presented in both a virtual environment and on-screen, to clarify the true value of VR as an effective renovation to the traditional paradigm.

In the present study, we aimed to further assess the utility of VR in TFP research by incorporating the same trauma film for both VR and on-screen conditions to enable direct comparison of implementing VR presentation of a trauma analogue within the traditional paradigm. The film used in the present study represents a realistic and plausible traumatic event of a car accident compared against the same film presented in a virtual environment. The film was provided by the Leicestershire Fire and Rescue Service (UK) and created as an educational tool to demonstrate the consequences of reckless driving and a typical first-response scene following a car accident. The film was created with actors playing the roles of the driver and passengers in the car, as well as the first-response ambulance and fire service. Due to the realistic nature of the trauma film and its compatibility with both on-screen and VR modality, the present study is able to provide a unique and direct exploration into the value of renovating the traditional TFP integrating a realistic, immersive trauma film in VR.

Emotional responses including anxiety and mood, as well as intrusion frequency and emotional characteristics of intrusions, were measured to assess and compare trauma responses between VR and on-screen presentation of the film. In alignment with Cuperus et al. (2017), self-reported personal involvement and realness was also measured to determine differences in feelings of presence between VR and on-screen conditions. Based on previous findings, we expected the VR presentation to evoke a greater sense of presence compared to on-screen presentation of the same traumatic film. We also hypothesised an increase in state anxiety and negative mood across both conditions with a greater increase expected following presentation in VR due to the known impact of greater sense of presence on affective response (Riva et al., 2007). Finally, on account of the two aforementioned comparable VR studies, one would expect the trauma film to generate equivalent frequency of intrusions regardless of film modality (VR or on-screen).

\section{Method}

\section{Participants}

A total of 66 undergraduate participants were recruited from the Psychology department at The University of Plymouth in return for course credit. Participants were informed of the graphic content of the film containing scenes of injury and informed consent was received from all participants prior to viewing the film. An a priori power analysis was conducted to generate an estimated sample size based on the difference in sense of presence scores between VR and on-screen conditions in a previous comparative study (Dibbets \& Schulte-Ostermann, 2015). With an alpha of .05 and power of .80, the projected sample size needed to generate the same large effect size ( $d=1.40$ ) was $N=20$ (ten in each condition), (Faul et al., 2009). However, as intrusion frequency was also measured, we over-recruited participants to each condition with the anticipation that some participants would not experience intrusions and/or comply with intrusion diary guidelines. After screening, 63 participants were included. A further two participants were excluded from analysis as they terminated the study before viewing the film. The final sample consisted of 61 participants randomly assigned to either VR $\left(N=31 ; M_{\text {age }}=20.7\right)$ or OnScreen (OS) $\left(N=30 ; M_{\text {age }}=20.8\right)$. Age of participants ranged from 18-52 years. The study was approved by the Ethical Committee of the Faculty of Health and Human Sciences at The University of Plymouth (17/18-805).

\section{Measures}

\section{Screening Questionnaire}

The screening tool incorporated was adopted from VR TFP research by Dibbets and Schulte-Ostermann (2015). The Jellinek-PTSD (J-PTSD) is a short self-report questionnaire consisting of four yes/no items pertaining to the four PTSD symptom clusters: re-experiencing; avoidance; hyper-arousal and negative affect (van Dam et al., 2013). Due to the nature of the film used representing a real-life trauma event, an additional question: 'Have you ever been 
personally involved in a car accident' was incorporated into screening. Only participants who responded negatively to all questions participated in the study.

\section{State Anxiety}

The Spielberger State Anxiety Inventory (STAI)-Short Form, (Marteau \& Bekker, 1992) was completed before and after the film to assess baseline measures as well as the impact of the film on participants' state anxiety. The STAIshort form is a brief six-item measure of state anxiety comprised of six questions requiring a response on a rating scale of 1-4 (Not at all to Almost Always). The STAI-short form shows satisfactory inter-item reliability with this sample at baseline $(\alpha=.68)$ and good inter-item reliability in post-film ratings $(a=.80)$.

\section{Mood}

Participants indicated their current mood on visual analogue rating scales ranging from zero (Not at all) to 100 (Extremely) for four different moods: Happy, Depressed, Anxious and Angry. These moods were selected in line with previous TFP research (Cuperus et al., 2017; Davis \& Clark, 1998). Mood ratings were completed both before and after the film to establish baseline ratings as well as the impact of the film on participants' mood.

\section{Sense of Presence}

Sense of presence was assessed post-film with the use of two statements regarding how participants felt whilst watching the trauma film: (1) / felt personally involved in the car accident, and (2) What happened somehow seemed real. Participants responded on a visual analogue scale that ranged from zero (not personally involved at all; did not feel real at all) to 100 (felt extremely real; extremely personally involved). These statements were adopted and modified from a post-film evaluation questionnaire by Cuperus et al. (2017) which compared VR and on-screen viewing of a trauma film. In an extension to the current study using the same trauma film with different outcome measures, these items were later endorsed against a validated measure of presence, the ITC-Sense of Presence Inventory (ITC-SOPI) (Lessiter et al., 2001). Both items produced a strong positive correlation with the presence measure from the ITC-SOPI, $(r=.70, p<.001)$.

\section{Intrusions}

Involuntary intrusions were logged in an online diary over the course of three days following the film. Participants received a daily email containing instructions of how to use the diary to record intrusions and a unique login link that directed them to their diary. Participants were instructed to login to their diary whenever they experienced an involuntary intrusion of the film using the link emailed; there were no limits on the number of times a participant could login to their diary over the study period. Once logged in, participants recorded the content of the intrusions experienced as well as rated the level of distress, unpleasantness, control and vividness of intrusions on four visual analogue scales ranging from 0 (low levels) to 100 (high levels of the specific attribute). Participants were also instructed to identify the modality of intrusions as being either 'visual', 'auditory' or 'both visual and auditory', as well as to report any possible trigger for the intrusions. Only intrusions containing a visual component were considered for analysis to exclude mere thoughts of the film and intrusions reported to have been triggered by the diary email were discounted from analysis. Participants were instructed to log in to the diary every day for three consecutive days following the trauma film regardless of intrusion experience, (i.e. participants who did not experience intrusions were instructed to input 'zero' into the intrusion number response box). Compliance with the intrusion diary was measured in a follow-up questionnaire emailed to participants on Day 4 (following completion of the intrusion diary).

\section{Trauma Film}

The Leicestershire Fire and Rescue Service (UK) provided the trauma film used. The film lasted 6:20 minutes and was created by attaching a $360^{\circ}$ camera rig to the headrest of the front passenger seat of a car. Within the car, a young male actor played the role of the distracted driver and two young female actors played the roles of distracting passengers. The film depicts erratic driving with the driver on his phone and interacting with the 
passengers. The scene ends when a tractor pulls out in front of the car, causing a collision. The film uses intermittent black screens with audio from the point of impact onwards to simulate loss of consciousness. In the trauma aftermath, the driver and one of the passengers are shown with life-threatening injuries, paramedics working to resuscitate them and the fire service removing the roof of the car. The scene ends with a paramedic tending to the passenger and the implied body of the participant in the front of the car (Figure 1).

Participants in the VR condition viewed the film using a HTC Vive VR headset and headphones. Participants were instructed to remain seated, as if embodying the front-seat passenger, but move their heads to navigate the scene and orientate their viewpoint. The film content is designed in such a way to draw attention from the viewer to different aspects of the scene with interaction towards the supposed body of the participant from other passengers and first-responders. Practice using the VR headset was provided prior to viewing the film.

Figure 1. Stills From the Trauma Film Showing the Distracted Driver and Aftermath of the Crash With First Responders Tending to Passengers and the Supposed Body of the Participant.
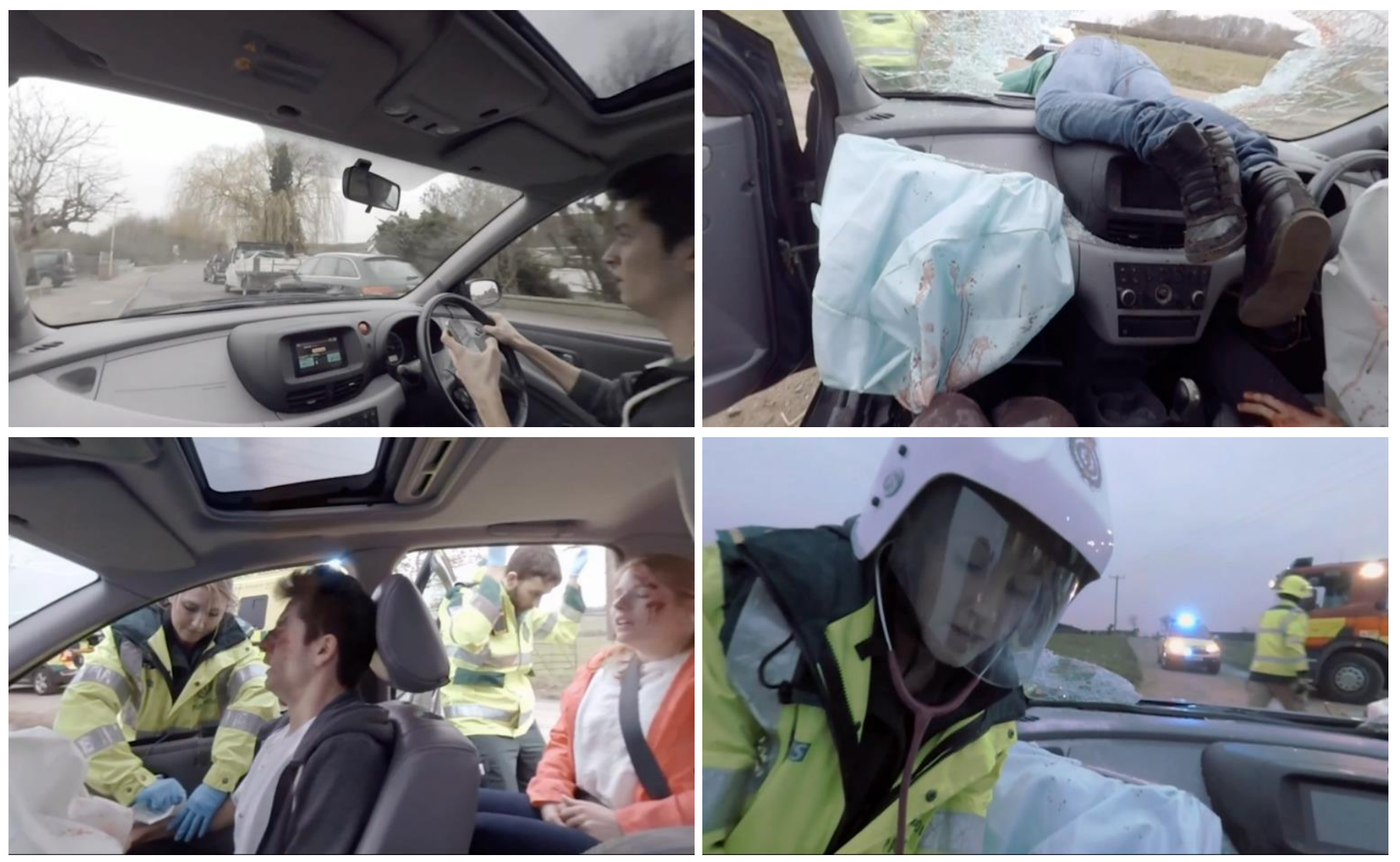

The film used in the OS condition was the same car accident film presented in the VR condition but with a forced perspective. Therefore, participants in the OS condition did not have the capacity to navigate the scene and instead viewed the film on a computer screen in front of them. The film was created by recording what is seen in the VR headset using screen capture in a way to ensure all main events in the film remained a main focal point. Participants wore headphones and were seated at a computer screen to watch the film on-screen.

\section{Procedure}

We used a mixed design with presentation modality as a between-subjects factor and participants randomly assigned to either view the trauma film in VR with a HTC Vive headset, or watch the same film on a computer screen in front of them (VR, OS). Pre-film to post-film changes in STAI and mood scores were within-subject factors (Figure 2).

Participants completed a pre-study screening questionnaire (see section Measures) and only participants who responded negatively to all five items from the questionnaire were eligible to take part. Participants were provided 
with a detailed information sheet with a disclaimer regarding the graphic content of the film containing scenes of injury.

Participants completed baseline questionnaires of self-report state anxiety and mood and were randomly allocated to either VR or OS condition. All participants were instructed to 'immerse completely in the film'. Those in the OS condition wore headphones and were prompted to press a 'Continue to film' button when ready to view the film on a computer screen positioned in front of them. In the VR condition, participants were provided with headphones and VR headset and were asked to signal to the researcher when ready to view the film. The researcher then started the film and viewed on-screen the participant's viewpoint to ensure participants were engaging with the virtual environment. At the end of the film, participants received instruction to remove the headset and completed the remaining scales.

Figure 2. Flowchart of Study Procedure.

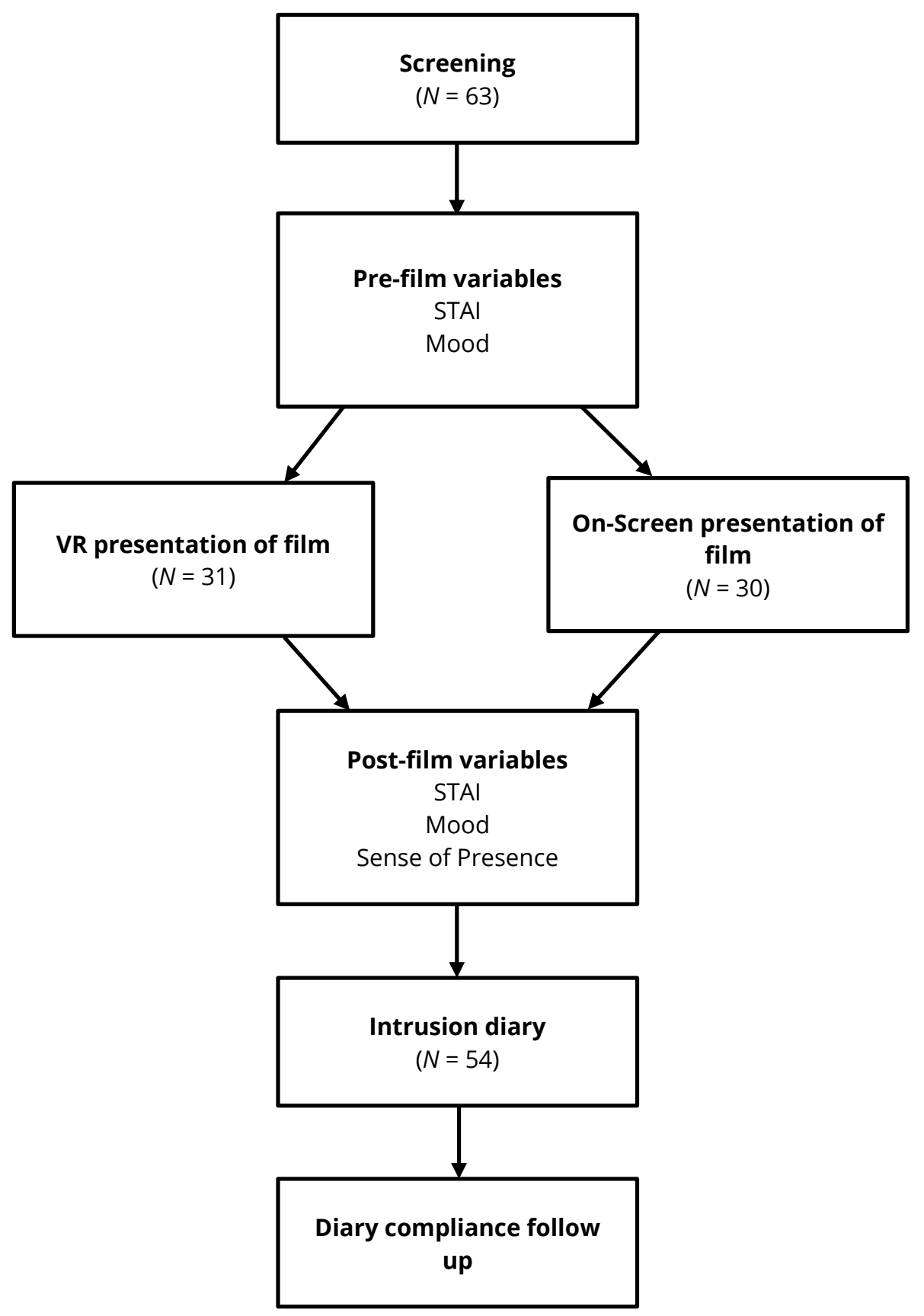

Following the film, all participants completed measures of state anxiety and mood to assess changes from baseline, as well as a sense of presence measure. Subsequently, participants were provided written and verbal instructions of how to use their diary to record any intrusions, defined as 'a memory or image which comes into awareness spontaneously' in accordance with previous definitions of intrusions (Cuperus et al., 2017). An automated email with a login link to the diary was sent to participants each day for three consecutive days beginning from 
the day after viewing the film. On the fourth day, participants were emailed a link to a short diary-compliance questionnaire, debrief and received course credit.

\section{Data Analyses}

To compare trauma responses between the VR and OS conditions, $2 \times 2$ repeated measures ANOVAs were conducted on pre- and post- film scores for state anxiety and mood as within-subjects factors and condition (VR or OS) as between-subjects factor. A 2x2 ANOVA was used to assess differences in sense of presence between conditions and an independent samples t-test was carried out to compare intrusion frequency. Finally, characteristics of intrusions were examined with a 2x4 ANOVA and regression analyses were conducted to generate predictive models of post-film distress, intrusion frequency and severity. Effect sizes were reported and the rejection criterion was set at $p<.05$ throughout.

\section{Results}

\section{Baseline Measures}

Mean scores from pre-film baseline measures for both conditions are provided in Table 1. There were no significant differences between VR and On-Screen (OS) conditions for pre-film state anxiety scores, $[F(1,59)=0.93$, $\left.p=.34, \eta^{2}=.02\right]$ or pre-film mood scores, $\left[F s(1,59)<2.05, p s>.16, \eta^{2}=.01\right]$.

Table 1. Mean Scores, Standard Deviations (SD) and Ranges for Pre- and PostFilm Measures for Both VR and OS Conditions.

\begin{tabular}{|c|c|c|c|c|c|c|}
\hline & \multicolumn{3}{|c|}{$\operatorname{VR}(N=31)$} & \multicolumn{3}{|c|}{ OS $(N=30)$} \\
\hline & Mean & $S D$ & Range & Mean & $S D$ & Range \\
\hline \multicolumn{7}{|l|}{ Pre-Film } \\
\hline State Anxiety & 9.39 & 2.0 & $6-14$ & 8.83 & 2.48 & $6-15$ \\
\hline Happy Mood & 72.23 & 14.40 & $50-99$ & 71.57 & 13.84 & $50-90$ \\
\hline Depressed Mood & 18.48 & 17.53 & $0-40$ & 15.87 & 17.24 & $0-95$ \\
\hline Anxious Mood & 7.48 & 11.86 & $0-58$ & 10.40 & 23.74 & $0-60$ \\
\hline Angry Mood & 1.03 & 2.32 & $0-10$ & 4.33 & 12.64 & $0-66$ \\
\hline \multicolumn{7}{|l|}{ Post-Film } \\
\hline State Anxiety & 15.65 & 3.44 & $7-21$ & 15.03 & 3.87 & $7-21$ \\
\hline Happy Mood & 45.06 & 20.95 & $0-85$ & 47.90 & 23.69 & $0-96$ \\
\hline Depressed Mood & 47.97 & 27.17 & $0-72$ & 35.37 & 29.84 & $0-71$ \\
\hline Anxious Mood & 25.32 & 24.81 & $0-93$ & 17.73 & 23.32 & $0-90$ \\
\hline Angry Mood & 14.32 & 22.65 & $0-70$ & 12.97 & 20.36 & $0-73$ \\
\hline
\end{tabular}

\section{State Anxiety}

Pre- and Post-film state anxiety mean scores for both VR and OS conditions are presented in Table 1. A 2x2 repeated measures ANOVA was conducted on pre-film and post-film STAI scores as within-subjects factors and condition (VR or OS) as the between-subjects factor to assess the impact of the trauma film on self-report state anxiety. The analysis revealed a main effect of time, $F(1,59)=205.08, p<.001, \eta^{2}=.78$, indicating an increase in state anxiety post-film, yet no main effect for condition, $F(1,59)=0.82, p=.37, \eta^{2}=.01$. There was also no interaction found between Condition $\times$ Time, $F(1,59)=0.004, p=.95, \eta^{2}<.001$.

\section{Mood}

Mean Pre- and Post-film mood scores for both VR and OS conditions are presented in Table 1. Analysis of each individual mood item revealed a main effect of time for each of the four moods measured, $F s(1,59)>19.87, p s<$ $.001, \eta^{2}>.26$, indicating a decrease in happiness and increase in anxious, depressed and angry mood following 
the film. Yet, no main effects for condition were found for any of the four moods, $F s(1,59)<2.15, p s>.15, \eta^{2}<.04$. Crucially, there were no significant interactions found between Condition $\times$ Time for any of the four mood scores, $F s(1,59)<3.72, p s>.06, \eta^{2}<.06$.

\section{Sense of Presence}

Differences in self-report sense of presence between the VR and OS conditions are presented in Figure 3. A 2x2 ANOVA revealed a statistically significant difference between conditions in feelings of being personally involved in the car accident, (VR: $M=59.45, S D=25.5 ; O S: M=35.67, S D=27.51$ ), $F(1,59)=12.27, p=.001, \eta^{2}=.17$, as well as the trauma event seeming real, (VR: $M=71.03, S D=22.87$; OS: $M=54.87, S D=29.91), F(1,59)=5.65, p=.02, \eta^{2}=$ .09. Indicating an increased sense of presence in the VR condition compared with the OS condition.

Figure 3. Mean Scores for Self-Report Sense of Presence for VR Condition ( $N=31)$ and OS Condition $(N=30)$.

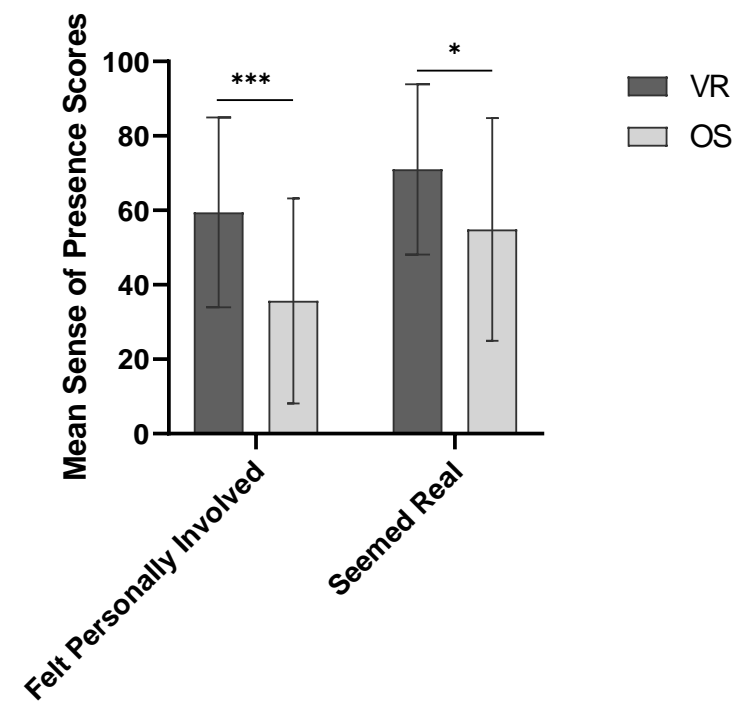

Sense of Presence Items

*** Significant at the .001 probability leve

* Significant at the .05 probability level

Note. Error bars indicate standard deviations.

\section{Intrusion Frequency}

Seven participants did not comply with the involuntary memory diary guidelines that instructed participants to log in to the diary every day for three consecutive days regardless of intrusion experience. Of these seven participants, three participants did not log in to the diary at all over the three days and four participants only complied with diary entry guidelines for two out of three days. Subsequently, these seven participants' data were removed from analysis and all consequent analysis was conducted on the remaining 54 participants, (27 participants in the VR condition and 27 in the OS condition). Follow-up revealed good compliance with the online diary with $92 \%$ of respondents reporting fairly or very confident in their accuracy of reporting each intrusion.

From the 54 participants who complied with the intrusion diary instructions, 30 participants reported at least one intrusion over the course of the following three days (VR Condition: $N=18$; OS Condition: $N=12$ ). The remaining 24 participants followed instruction by logging in to their online diary each day but reported zero intrusions. Including all participants who complied with intrusion diary guidelines $(N=54)$, a Mann-Whitney $U$ test was conducted to compare intrusion frequency between conditions as a non-parametric alternative to an Independent Samples t-test due to data not being normally distributed. Analysis revealed no significant differences in mean number of intrusions reported between participants in the VR condition, $(M=1.33, S D=1.30)$ and those in the OS condition, $(M=1.52, S D=2.56), U=310.50, p=.32$. Removal of two outliers (intrusion scores of 9 ) restored normality of distribution but did not alter the outcome of the analysis. 


\section{Intrusion Characteristics}

As well as number of intrusions, participants were required to report emotionality of their intrusions experienced on four measures including: Distress; Control; Unpleasantness and Vividness. Subsequently, only participants who had recorded at least one intrusion were included in the following analysis (VR condition: $N=18$; OS condition: $N$ $=12$. . Differences in intrusion emotionality between conditions are presented in Figure 4. A 2x4 ANOVA revealed no significant differences between conditions in the level of distress, control or unpleasantness of intrusions, $F s(1,28)<1.02$, $p s>.32$. However, the groups differed in mean self-reported vividness of intrusions, $F(1,28)=4.58$, $p=.04, d=0.80$, with those from the VR condition reporting greater vividness of intrusions $(M=57.11, S D=25.09)$ compared with those in the OS condition $(M=37.50, S D=23.81)$. However, reliance on data from participants who experienced intrusions only, means that this analysis was performed on relatively small sample sizes for each condition. Therefore, despite a large effect size for difference in intrusion vividness, no firm conclusions can be drawn due to potential issues of low power.

Figure 4. Mean Scores for Self-Report Intrusion Characteristics for VR $(N=18)$ and On Screen $(N=12)$ Conditions.

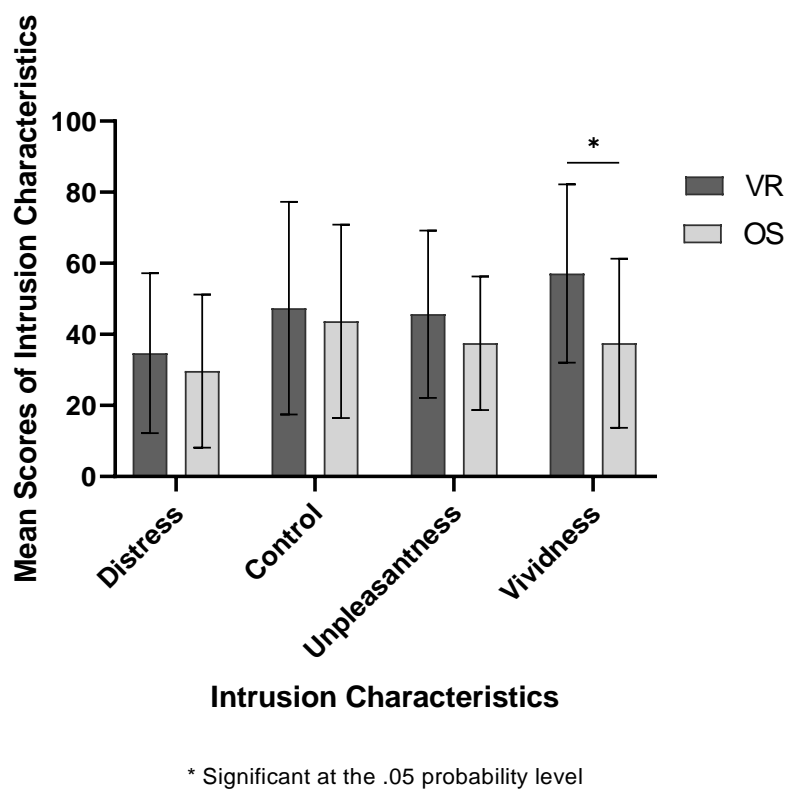

Note. Error bars indicate standard deviations.

\section{Regression Analyses}

Regression analyses were conducted to test the extent to which sense of presence can explain post-film distress, later intrusion frequency or emotionality. As the data revealed no significant differences between conditions in pre-film baseline measures or intrusion frequency, participants in both VR and OS conditions were combined for analysis. Prior to performing regression analyses, the relevant assumptions for singularity, normality, linearity and individual outliers were tested and satisfied upon removal of two participants who reported nine intrusions (Field, 2018). Linear regression analysis revealed combined sense of presence scores independently significantly predicted intrusion frequency, $F(1,50)=4.22, p=.05, R^{2}=.08$, and level of distress associated with intrusions, $F(1,26)=6.84, p=.02, R^{2}=.21$. No other characteristics of intrusions measured were significantly associated with sense of presence. However, analysis with all participants revealed that sense of presence did significantly predict post-film STAI scores, $F(1,50)=8.20, p=.006, R^{2}=.14$; post-film depressed mood $F(1,59)=12.45, p=.001, R^{2}=.17$, and post-film anxious mood $F(1,59)=15.74, p<.001, R^{2}=.21$. These analyses indicate that a greater sense of presence from an analogue trauma predict greater state anxiety, depressed and anxious mood as well as a higher frequency of intrusions and self-report distress from intrusions.

\section{Discussion}

This study aimed to build upon preliminary research investigating the value of incorporating a virtual trauma analogue into the traditional film paradigm. The novel element of this research lies in the same realistic film used 
for both VR and on-screen presentation to allow for a direct assessment of the utility of a virtual trauma analogue. The trauma film used in this study evoked an increase in negative mood and state anxiety comparable to previously published TFP research, (Cuperus et al., 2017). The film also elicited involuntary intrusions over the following three days, yet an important limitation to note is that the average frequency of intrusions was lower ( $M$ $=1.43$ ) than those reported in previous TFP studies incorporating a 3-day diary which range from $M=2.26$ to $M=$ 3.42 (Luo et al., 2013; Verwoerd et al., 2012). However, for the purpose of generating a direct comparison between VR and OS modality of trauma film presentation, the actual frequency of intrusions is less significant than the differences in responses between the two conditions. The comparatively lower number of intrusions may be as a result of the film appearing less shocking compared with real-life footage of road accidents used in previous TFP research. The staged nature of the film prevented footage of an actual collision and instead presented intermittent black screens to imply collision and loss of consciousness of the passenger (viewer). Yet, in order for immersive and realistic VR to be used appropriately in TFP research, filming requires the use of a $360^{\circ}$ camera and is therefore limited to staged trauma events (as opposed to dash-cam footage of a real-life road accident). However, this should not deter future research from incorporating VR. A review of trauma film genres suggests clips from feature films depicting staged scenes of violence generate more intense stress reactions than compilations of footage from real-events, (Weidmann et al., 2009). This suggests that film genre may be more influential over trauma responses than the staged nature of the film.

When considering the effectiveness of incorporating VR into TFP research, the findings indicate that VR is comparable to on-screen presentation of a trauma analogue for post-trauma measures of state anxiety and the later development of distressing and unpleasant intrusive memories. This suggests that presenting a trauma film in VR is equally effective at inducing such post-trauma responses as presentation of the same analogue trauma seen on-screen. However, intrusions after VR appear to be more vivid than those after on-screen presentation, a finding that contradicts previous comparable research (Cuperus et al., 2017). It seems likely to assume that this disparity may be due to the animated nature of the virtual environments used in previous research eliciting intrusions that are less vivid than their realistic, on-screen alternative. The use of identical film content in the current study eradicates such nuances and posits a VR-modality-specific contributing factor to evoke enhanced vividness of intrusions. Greater vividness of intrusions following VR presentation may originate from the virtual environment providing an egocentric first-person view of the trauma scene eliciting self-referent memories that are more akin to autobiographical memories of a real life event than viewing the scene on-screen (Rubin \& Umanath, 2015). However, due to comparatively low numbers of participants in each condition who experienced intrusions, the observed findings from this study can offer preliminary evidence of increased intrusion vividness following VR presentation, but cannot draw firm conclusions. Moreover, future research incorporating VR should question the clinical relevance of enhanced vividness of intrusions when applied to PTSD symptoms following reallife trauma due to the recognised significance of distress caused by intrusions, (rather than intrusion vividness) as a predictive factor of PTSD severity, (Michael et al., 2005; Reger et al., 2019).

Those who had viewed the trauma film in VR experienced a greater 'sense of presence': a finding reflected in previous comparable research (Cuperus et al., 2017; Dibbets \& Schulte-Ostermann, 2015). This finding may reflect the increased interactivity of freedom of viewpoint experienced by participants in the VR condition. The use of VR in clinical settings, such as exposure therapy for anxiety disorders, is based on the assumption that the increased feeling of presence, i.e. the sense of actually being there, acts as a catalyst to generate anxiety and distress in clinical virtual environments (Alsina-Jurnet et al., 2011). The findings from the regression analyses presented in this study support this theory and demonstrate that, regardless of medium, a greater sense of presence is predictive of greater emotional response to an analogue trauma in terms of increased state anxiety, depressed and anxious mood. Additionally, greater sense of presence was also indicative of later intrusion frequency and distress experienced from intrusions. These findings are in line with other VR research that reports sense of presence as a mediating factor between the media experience and affective response induced by it (Riva et al., 2007). This is of particular relevance in TFP research aiming to produce an analogue for a real life trauma event. If a trauma film cannot evoke a feeling of presence, the emotional response may be low regardless of the stressful content of the film itself. Equally, trauma films that induce a higher degree of involvement, such as having a straight story line as opposed to a series of different scenes, are associated with a more intense stress response (Weidmann et al., 2009). Future research may consider exploring which elements of presence may contribute to enhanced affective response following an analogue trauma. 
Car accident footage is commonly used in TFP research as it affords a reflection of a real-event involving actual or threatened death and serious injury in accordance with defined trauma events in the context of PTSD diagnosis (American Psychiatric Association, 2013). The use of VR offers self-paced exploration of the trauma scene as the participant is able to manipulate their viewing perspective as if they were a passenger in the car themselves. This increased interactivity and engagement, albeit limited to head movements in the current study, increases immersion at the expense of control over stimulus presentation as each participant's view of the film would be slightly different (Rizzo \& Shilling, 2017). This is partially controlled for in the current study as the scenes are created in a way to manipulate the observer perspective, yet future research would be wise to take into account the cost of participant-controlled interactions and balance interactivity with controllability. Presently, the difficulties associated with the availability of $360^{\circ}$ footage and ability to produce realistic virtual environments causes a barrier for the appropriate integration of VR into TFP research. The current findings present little impact of VR on intrusion frequency or distress, which are typically the main outcome measures in TFP studies (Weidmann et al., 2009). Therefore, the increased sense of presence in VR should not necessarily override the caveat of less realistic and credible film footage.

The film used in the current study presents a real-life trauma scenario to expand on previous TFP research incorporating programmed virtual environments. The use of programmed simulations has been effective as a therapeutic medium to deliver exposure therapy for PTSD, particularly in contexts whereby actual exposure is not possible (e.g. military experience), (Rizzo et al., 2010). Programmed virtual environments have also been effective in clinical assessments of PTSD in an attempt to develop an objective measure of physiological response to simulated trauma scenes (Costanzo et al., 2014). However, when developing an analogue for a real life trauma event amongst a healthy population, the credibility and relatability of the analogue trauma is significant for generalisability of TFP research to trauma symptoms following events in the real world (Rovira et al., 2009). Incorporating VR into trauma film paradigms presents an opportunity to provide ecologically relevant and realistic simulations of stressful environments as an analogue for a real-life traumatic event. Yet this application of realistic VR film footage should be approached with strong consideration of the ethical implications for participants. The film used in the current study was originally created as a deterrent for reckless driving and did not contain highly aversive content. Data from the intrusion diaries suggests most participants experienced a decrease in intrusion frequency over the course of the three days with the majority of intrusions reported within the first day after watching the film. However, future research intending on increasing realism with VR trauma films should consider potential long-term consequences of immersive trauma analogue exposure.

Employing an online intrusion diary as opposed to paper-pen diaries coincides with the aspiration to renovate the traditional TFP methodology. Participants received a login link via email to their personal online intrusion diary that could be accessible from smartphones, tablet or computer at any time point throughout the study period. Whereas paper-pen diaries could be mislaid or completed retrospectively and therefore fail to provide an accurate representation of intrusion frequency, an online diary offers sustained accessibility and record of participant engagement and has been successfully incorporated into previous TFP research, (e.g. Ball \& Brewin, 2012; Kamboj et al., 2014). The potential limitation of this method surrounds the possibility of the email containing login details for the diary causing a trigger to the film. However, as part of the reporting process, participants were instructed to identify triggers that may have evoked intrusions and any reports containing details of the email being the trigger were discounted from analysis. Additionally, only intrusions with a visual component were considered for analysis to eradicate the occurrence of mere thoughts of the experiment, which would likely arise from reading the email. We concluded that an email would cause no more of a trigger than the presence of a paper-pen diary and so incorporated an online diary into the study design and achieved high participant compliance rates at follow up. Future studies may consider a comparison in methodology of intrusion recording, yet the use of online diaries could offer a more sustainable alternative to the traditional paradigm that better reflects a more technologically advanced population.

\section{Conclusions}

Presenting an analogue trauma in a virtual environment can benefit TFP research by increasing engagement with the trauma scene making the experience seem more real and enhance feelings of being personally involved. Although in the current study there are no direct differences in intrusion frequency or distress between VR an onscreen film presentation, there is evidence that an enhanced sense of presence, regardless of film modality, results 
in greater frequency and distress from later intrusions. Consequently, future research should consider the immersive nature of films used as an analogue for a traumatic event.

\section{Acknowledgements}

We would like to give special thanks to Mr. Paul Speight, Watch Manager at Leicestershire Fire and Rescue Service, for providing the road safety virtual reality film used in our research.

\section{References}

Alsina-Jurnet, l., Gutiérrez-Maldonado, J., \& Rangel-Gómez, M.-V. (2011). The role of presence in the level of anxiety experienced in clinical virtual environments. Computers in Human Behavior, 27(1), 504-512.

https://doi.org/10.1016/j.chb.2010.09.018

American Psychiatric Association. (2013). Diagnostic and statistical manual of mental disorders: DSM-5 ${ }^{\mathrm{TM}}$ (5th ed.). American Psychiatric Publishing, Inc.

Ball, S. C., \& Brewin, C. R. (2012). The effect of rumination on intrusive images and mood: An experimental investigation using the trauma film paradigm. Journal of Experimental Psychopathology, 3(2), 279-309. https://doi.org/10.5127/jep.019511

Beidel, D. C., Frueh, B. C., Neer, S. M., Bowers, C. A., Trachik, B., Uhde, T. W., \& Grubaugh, A. (2019). Trauma management therapy with virtual-reality augmented exposure therapy for combat-related PTSD: A randomized controlled trial. Journal of Anxiety Disorders, 61, 64-74. https://doi.org/10.1016/j.janxdis.2017.08.005

Boskovic, I., Dibbets, P., Bogaard, G., Hope, L., Jelicic, M., \& Orthey, R. (2019). Verify the scene, report the symptoms: Testing the Verifiability Approach and SRSI in the detection of fabricated PTSD claims. Legal and Criminological Psychology, 24(2), 241-257. https://doi.org/10.1111/lcrp.12149

Botella, C., Serrano, B., Baños, R. M., \& Garcia-Palacios, A. (2015). Virtual reality exposure-based therapy for the treatment of post-traumatic stress disorder: A review of its efficacy, the adequacy of the treatment protocol, and its acceptability. Neuropsychiatric Disease and Treatment, 11, 2533-2545. https://doi.org/10.2147/NDT.S89542

Candel, I., \& Merckelbach, H. (2004). Peritraumatic dissociation as a predictor of post-traumatic stress disorder: A critical review. Comprehensive Psychiatry, 45(1), 44-50. https://doi.org/10.1016/j.comppsych.2003.09.012

Carl, E., Stein, A. T., Levihn-Coon, A., Pogue, J. R., Rothbaum, B., Emmelkamp, P., Asmundson, G. J. G., Carlbring, P., \& Powers, M. B. (2019). Virtual reality exposure therapy for anxiety and related disorders: A meta-analysis of randomized controlled trials. Journal of Anxiety Disorders, 61, 27-36. https://doi.org/10.1016/j.janxdis.2018.08.003

Chou, C.-Y., La Marca, R., Steptoe, A., \& Brewin, C. R. (2014). Biological responses to trauma and the development of intrusive memories: An analog study with the trauma film paradigm. Biological Psychology, 103, 135-143. https://doi.org/10.1016/j.biopsycho.2014.08.002

Costanzo, M. E., Leaman, S., Jovanovic, T., Norrholm, S. D., Rizzo, A. A., Taylor, P., \& Roy, M. J. (2014). Psychophysiological response to virtual reality and subthreshold posttraumatic stress disorder symptoms in recently deployed military. Psychosomatic Medicine, 76(9), 670-677.

https://doi.org/10.1097/PSY.0000000000000109

Cuperus, A. A., Klaassen, F., Hagenaars, M. A., \& Engelhard, I. M. (2017). A virtual reality paradigm as an analogue to real-life trauma: Its effectiveness compared with the trauma film paradigm. European Journal of Psychotraumatology, 8(Suppl. 1), Article 1338106. https://doi.org/10.1080/20008198.2017.1338106 
Cuperus, A. A., Laken, M., van den Hout, M. A., \& Engelhard, I. M. (2016). Degrading emotional memories induced by a virtual reality paradigm. Journal of Behavior Therapy and Experimental Psychiatry, 52, 45-50.

https://doi.org/10.1016/j.jbtep.2016.03.004

Davis, M. I., \& Clark, D. M. (1998). Predictors of analogue post-traumatic intrusive cognitions. Behavioural and Cognitive Psychotherapy, 26(4), 303-314. https://doi.org/10.1017/S1352465898264022

Dibbets, P. (2020). A novel virtual reality paradigm: Predictors for stress-related intrusions and avoidance behavior. Journal of Behavior Therapy and Experimental Psychiatry, 67, Article 101449.

https://doi.org/10.1016/j.jbtep.2019.01.001

Dibbets, P., \& Schulte-Ostermann, M. A. (2015). Virtual reality, real emotions: A novel analogue for the assessment of risk factors of post-traumatic stress disorder. Frontiers in Psychology, 6, Article 681.

https://doi.org/10.3389/fpsyg.2015.00681

Faul, F., Erdfelder, E., Buchner, A., \& Lang, A.-G. (2009). Statistical power analyses using G* Power 3.1: Tests for correlation and regression analyses. Behavior Research Methods, 41(4), 1149-1160.

https://doi.org/10.3758/BRM.41.4.1149

Field, A. (2018). Discovering statistics using IBM SPSS statistics (5th ed.). Sage.

Halligan, S. L., Clark, D. M., \& Ehlers, A. (2002). Cognitive processing, memory, and the development of PTSD symptoms: Two experimental analogue studies. Journal of Behavior Therapy and Experimental Psychiatry, 33(2), 73-89. https://doi.org/10.1016/S0005-7916(02)00014-9

Holmes, E. A., Brewin, C. R., \& Hennessy, R. G. (2004). Trauma films, information processing, and intrusive memory development. Journal of Experimental Psychology: General, 133(1), 3-22. https://doi.org/10.1037/00963445.133.1.3

Horowitz, M. J. (1969). Psychic trauma: Return of images after a stress film. Archives of General Psychiatry, 20(5), 552-559. https://doi.org/10.1001/archpsyc.1969.01740170056008

lyadurai, L., Blackwell, S. E., Meiser-Stedman, R., Watson, P. C., Bonsall, M. B., Geddes, J. R., Nobre, A. C., \& Holmes, E. A. (2018). Preventing intrusive memories after trauma via a brief intervention involving Tetris computer game play in the emergency department: a proof-of-concept randomized controlled trial. Molecular Psychiatry, 23(3), 674-682. https://doi.org/10.1038/mp.2017.23

Kamboj, S. K., Oldfield, L., Loewenberger, A., Das, R. K., Bisby, J., \& Brewin, C. R. (2014). Voluntary and involuntary emotional memory following an analogue traumatic stressor: The differential effects of communality in men and women. Journal of Behavior Therapy and Experimental Psychiatry, 45(4), 421-426.

https://doi.org/10.1016/j.jbtep.2014.05.001

Laposa, J. M., \& Rector, N. A. (2012). The prediction of intrusions following an analogue traumatic event: Peritraumatic cognitive processes and anxiety-focused rumination versus rumination in response to intrusions. Journal of Behavior Therapy and Experimental Psychiatry, 43(3), 877-883.

https://doi.org/10.1016/j.jbtep.2011.12.007

Lessiter, J., Freeman, J., Keogh, E., \& Davidoff, J. (2001). A cross-media presence questionnaire: The ITC-Sense of Presence Inventory. Presence: Teleoperators and Virtual Environments, 10(3), 282-297.

https://doi.org/10.1162/105474601300343612

Loucks, L., Yasinski, C., Norrholm, S. D., Maples-Keller, J., Post, L., Zwiebach, L., Fiorillo, D., Goodlin, M., Jovanovic, T., Rizzo, A. A., \& Rothbaum, B. O. (2019). You can do that?!: Feasibility of virtual reality exposure therapy in the treatment of PTSD due to military sexual trauma. Journal of Anxiety Disorders, 61, 55-63.

https://doi.org/10.1016/j.janxdis.2018.06.004 
Luo, P., Jiang, Y., Dang, X., Huang, Y., Chen, X., \& Zheng, X. (2013). Effects of different forms of verbal processing on the formation of intrusions. Journal of Traumatic Stress, 26(2), 288-294. https://doi.org/10.1002/jts.21800

Marteau, T. M., \& Bekker, H. (1992). The development of a six-item short-form of the state scale of the Spielberger State-Trait Anxiety Inventory (STAI). British Journal of Clinical Psychology, 31(3), 301-306. https://doi.org/10.1111/j.2044-8260.1992.tb00997.x

Michael, T., Ehlers, A., Halligan, S. L., \& Clark, D. M. (2005). Unwanted memories of assault: What intrusion characteristics are associated with PTSD? Behaviour Research and Therapy, 43(5), 613-628.

https://doi.org/10.1016/j.brat.2004.04.006

Reger, G. M., Smolenski, D., Norr, A., Katz, A., Buck, B., \& Rothbaum, B. O. (2019). Does virtual reality increase emotional engagement during exposure for PTSD? Subjective distress during prolonged and virtual reality exposure therapy. Journal of Anxiety Disorders, 61, 75-81. https://doi.org/10.1016/j.janxdis.2018.06.001

Riva, G., Mantovani, F., Capideville, C. S., Preziosa, A., Morganti, F., Villani, D., Gaggioli, A., Botella, C. \& Alcañiz, M. (2007). Affective interactions using virtual reality: The link between presence and emotions. CyberPsychology \& Behavior, 10(1), 45-56. https://doi.org/10.1089/cpb.2006.9993

Rizzo, A., Buckwalter, J. G., John, B., Newman, B., Parsons, T., Kenny, P., \& Williams, J. (2012). STRIVE: Stress Resilience In Virtual Environments: A pre-deployment VR system for training emotional coping skills and assessing chronic and acute stress responses. Studies in Health Technology and Informatics, 173, 379-385. http://doi.org/10.3233/978-1-61499-022-2-379

Rizzo, A. S., Difede, J., Rothbaum, B. O., Reger, G., Spitalnick, J., Cukor, J., \& Mclay, R. (2010). Development and early evaluation of the Virtual Iraq/Afghanistan exposure therapy system for combat-related PTSD. Annals of the New York Academy of Sciences, 1208(1), 114-125. https://doi.org/10.1111/j.1749-6632.2010.05755.x

Rizzo, A. S., \& Shilling, R. (2017). Clinical virtual reality tools to advance the prevention, assessment, and treatment of PTSD. European Journal of Psychotraumatology, 8(Suppl. 5), Article 1414560.

https://doi.org/10.1080/20008198.2017.1414560

Rovira, A., Swapp, D., Spanlang, B., \& Slater, M. (2009). The use of virtual reality in the study of people's responses to violent incidents. Frontiers in Behavioral Neuroscience, 3, Article 59.

https://doi.org/10.3389/neuro.08.059.2009

Rubin, D. C., \& Umanath, S. (2015). Event memory: A theory of memory for laboratory, autobiographical, and fictional events. Psychological Review, 122(1), 1-23. https://doi.org/10.1037/a0037907

Schöne, B., Wessels, M., \& Gruber, T. (2019). Experiences in virtual reality: A window to autobiographical memory. Current Psychology, 38(3), 715-719. http://doi.org/10.1007/s12144-017-9648-y

Schweizer, T., Renner, F., Sun, D., Becker-Asano, C., \& Tuschen-Caffier, B. (2019). Cognitive processing and regulation modulates analogue trauma symptoms in a virtual reality paradigm. Cognitive Therapy and Research, 43(1), 199-213. https://doi.org/10.1007/s10608-018-9967-9

Schweizer, T., Renner, F., Sun, D., Kleim, B., Holmes, E. A., \& Tuschen-Caffier, B. (2018). Psychophysiological reactivity, coping behaviour and intrusive memories upon multisensory Virtual Reality and Script-Driven Imagery analogue trauma: A randomised controlled crossover study. Journal of Anxiety Disorders, 59, 42-52.

https://doi.org/10.1016/j.janxdis.2018.08.005

Schweizer, T., Schmitz, J., Plempe, L., Sun, D., Becker-Asano, C., Leonhart, R., \& Tuschen-Caffier, B. (2017). The impact of pre-existing anxiety on affective and cognitive processing of a Virtual Reality analogue trauma. PloS One, 12(12), Article e0190360. https://doi.org/10.1371/journal.pone.0190360 
van Dam, D., Ehring, T., Vedel, E., \& Emmelkamp, P. M. G. (2013). Screening for posttraumatic stress disorder in civilian substance use disorder patients: Cross-validation of the Jellinek-PTSD screening questionnaire. Journal of Substance Abuse Treatment, 44(1), 126-131. https://doi.org/10.1016/j.jsat.2012.03.005

Verwoerd, J., Wessel, I., \& De Jong, P. J. (2012). Fewer intrusions after an attentional bias modification training for perceptual reminders of analogue trauma. Cognition and Emotion, 26(1), 153-165.

https://doi.org/10.1080/02699931.2011.563521

Weidmann, A., Conradi, A., Gröger, K., Fehm, L., \& Fydrich, T. (2009). Using stressful films to analyze risk factors for PTSD in analogue experimental studies - which film works best? Anxiety, Stress \& Coping, 22(5), 549-569.

https://doi.org/10.1080/10615800802541986

\section{Correspondence to:}

Grace Baptie

School of Psychology

University of Plymouth

PL4 8AA

United Kingdom

Email: gracebaptie(at)gmail.com

Editorial record: First submission received on August 28, 2019. Revisions received on April 13, 2020 and December 2, 2020. Accepted for publication on December 10, 2020.

Editor in charge: Alexander Schouten

\section{About Authors}

Grace Baptie is completing her PhD in Psychology. Her research interests are on predisposing factors for the development of trauma symptoms, with particular focus on traumatic childbirth and the significance of factors such as support, control, dissociation and alexithymia.

Jackie Andrade is a professor of psychology at the University of Plymouth. Her research broadly covers the interplay between cognition and emotion, including psychological models of trauma memory, motivation, and craving.

Alison M. Bacon is Associate Professor in Psychology at University of Plymouth. Her research interests are personality and psychological impacts on health (particularly physical health) and the biopsychosocial approach to understanding health and behaviours.

Alyson Norman is a Lecturer in Psychology at University of Plymouth, School of Psychology. She runs the MSc Clinical Psychology programme and works privately as a psychotherapist. Her research interests include working with people with long term conditions, particularly those with appearance-altering conditions and brain injuries. 